\title{
Prognosen in der Hydrogeologie - Kristallkugel oder fundierte Wissenschaft?
}

\author{
Thomas Himmelsbach ${ }^{1}$ Christoph Neukum
}

Eingegangen: 11. Juni 2018 / Online publiziert: 4. Juli 2018

๑) Springer-Verlag GmbH Deutschland, ein Teil von Springer Nature 2018

Der globale Wandel mit seinen Herausforderungen durch den Klimawandel und die demographische Entwicklung wirkt sich weltweit auf die Georessource Grundwasser aus, die gleichermaßen Wirtschaftsgut und Lebensgrundlage ist. In Anbetracht des wachsenden Nachfragedrucks aus Landwirtschaft, Industrie und Wasserversorgung erlangen die Aspekte, wie qualitative Beschaffenheit und nachhaltige Bewirtschaftung, sowie die Neubildung und der Schutz der verfügbaren Grundwasserressourcen eine in die Zukunft gerichtete, zentrale Bedeutung. Es wird in Zukunft daher nicht mehr ausreichen, nur den Status Quo zu beschreiben, sondern es müssen belastbare Szenarien entwickelt und hieraus Prognosen abgeleitet werden, welche die bevorstehende qualitative und quantitative Entwicklung der Grundwasserressourcen zum Ziel haben. Derartige Prognosen beruhen in der Mehrzahl auf den Ergebnissen numerischer Modelle, sei es nun die Entwicklung der klimatischen Bedingungen und deren Rückkopplung über die Grundwasserneubildung auf die Grundwasserressourcen als Ganzes oder über die hydrologischen Abflussbedingungen infolge sich verändernder Niederschläge. Diese Modelle unterstellen häufig, dass sich die Grundwasserneubildung mehr oder weniger linear zum Niederschlag eines Jahres verhält. Aus unseren Untersuchungen in semiariden Gebieten des südlichen Afrikas wissen wir hingegen, dass die Grundwasserneubildung in starkem Maße von vorhandenem Bodenwasser sowie Intensität und Dauer einzelner Niederschlagsereignisse abhängt. Auf Deutschland übertragen könnten die sich ändernden klimatischen Bedingungen in manchen Gegenden zu einer deutlich reduzierten Grundwasserneubildung, in anderen zu einer stärkeren Neubildung führen. Im Umkehrschluss würde dies bedeuten, dass die Landwirt-

Thomas Himmelsbach

thomas.himmelsbach@bgr.de

$\triangle$ Christoph Neukum christoph.neukum@bgr.de

1 Bundesanstalt für Geowissenschaften und Rohstoffe, Stilleweg 2, 30655 Hannover, Deutschland schaft entweder extensiv betrieben bzw. die Feldberegnung intensiviert werden müsste oder dass es zu Vernässungen kommt. Die Zeitskala auf der sich Änderungen derartiger Prozesse abspielen ist bis dato unbekannt.

Ein weiterer, in die gleiche Richtung gehender Aspekt, betrifft Reaktionsfronten im Untergrund. Durch den massenhaften Import von Futtermitteln aus dem Ausland für die Viehzucht und der sich anschließenden Verwendung der Gülle als Dünger, entsteht ein sehr hoher Nitrateintrag in die Böden und Grundwasserleiter. Hierdurch kann die hinlänglich bekannte Reduktionskapazität des Untergrunds im Laufe der Zeit überbeansprucht werden und es lokal zu einem plötzlichen Durchbruch von Nitratfronten zu den Trinkwasserbrunnen kommen. Auch wenn aufgrund vielfältiger Untersuchungen in den letzten Dekaden die zugrundeliegende Kinetik weitgehend bekannt ist, so ist die jetzt innewohnende Dynamik und die zur Verfügung stehende Zeitspanne bis zur Erschöpfung der lokalen Reaktionskapazität hingegen nur schwer einschätzbar.

Die grundlegende Frage, die sich hiermit stellt ist, ob es eine gleichbleibende Änderung von Umwelt- und Randbedingungen gibt oder ob in diesem Zusammenhang sogenannte Tipping-Points zu erwarten sind, ab deren Auftreten sich zuvor scheinbar stabile Systeme unerwartet und schnell in eine bis dato unerwartete Richtung entwickeln.

Durch langjährige Beobachtungsreihen von bodenhydrologischen und hydrogeochemischen Daten sind wir in der Lage die Entwicklung von Stoffeinträgen (z.B. Versauerung, Nitrat, Spurenstoffe) und deren Wechselwirkungen mit dem Grundwasserleiter abzuleiten und diese Informationen in prognostische reaktive Transportmodelle umzusetzen. Die Verfügbarkeit von langjährigen Datenreihen ermöglicht es, Langzeitprognosen mit entsprechenden Modellen zuverlässiger ableiten zu können. Dementsprechend bedeutend ist die kontinuierliche Erhebung und zielgerichtete Interpretation von Daten.

Für unsere Disziplin ist es wichtig, bei der Ausarbeitung von hydrogeologischen Prognosen stets die aktuellen methodischen Grenzen zu berücksichtigen und diese im Sinne einer zukunftsfähigen Wissenschaft weiterzuentwickeln. 
Insbesondere der Kommunikation von Unsicherheiten in der Prognosefähigkeit unserer Daten und Modelle ist im Rahmen der Beratung von Gesellschaft und Politik ausreichend Platz einzuräumen, um nicht missverstanden zu werden und dadurch Vertrauen zu verspielen. Wir sind daher aufgefordert, verstärkt Anstrengungen zu unternehmen, be- stehende Methoden der Datenerhebung, deren Auswertung und Interpretation sowie das dahinterstehende Prozessverständnis und die darauf aufbauenden Simulationstechniken zu verbessern. Dies alles ist Grundlage einer verbesserten, in der Zukunft immer bedeutsamer werdenden Prognosefähigkeit unserer Methoden. 